\title{
Visual short-term memory is not improved by training
}

\author{
INGRID R. OLSON \\ University of Pennsylvania, Philadelphia, Pennsylvania \\ and \\ YUHONG JIANG \\ Harvard University, Cambridge, Massachusetts
}

\begin{abstract}
A critical question in visual working or short-term memory (VSTM) research is whether the ability to remember briefly presented visual stimuli can be increased. Here we test whether VSTM for locations and shapes is improved by training that allows one to utilize another memory system, visual longterm memory (VLTM). Training was done by repeatedly presenting a subset of memory displays, creating long-term memory traces for these displays. Surprisingly, VSTM performance for repeated displays was not higher than for nonrepeated ones, even though participants recognized repeated displays on a forced-choice test given at the end of the experiment. We suggest that the fidelity of information held by VLTM is inferior to that of information held by VSTM and thus provides no additional benefit over what is extracted on the fly by VSTM.
\end{abstract}

Visual working or short-term memory (VSTM) is used to retain visual information for up to a few seconds after a visual display is no longer in sight (Logie, 1995). It maintains a sense of continuity in a constantly changing visual environment, by holding visual information in memory between discrete saccades of the eye (Irwin, 1992). VSTM is highly limited in capacity: only approximately four simple objects such as colored circles (Luck \& Vogel, 1997; Raffone \& Wolters, 2001), letters (Pashler, 1988), and six spatial locations (Irwin, 1992; Jiang, Olson, \& Chun, 2000) can be retained. Items in VSTM are represented allocentrically, as a visual configuration encoded with reference to one another (Bor, Duncan, \& Owen, 2001; Jiang et al., 2000; Santa, 1977).

The small capacity of VSTM, four items or six locations, varies little with differences in features such as colors, orientations, or sizes (Vogel, Woodman, \& Luck, 2001). Whether or not the items are familiar has little effect (e.g., for faces; Buttle \& Raymond, 2003) or none at all (e.g., for letters; Pashler, 1988). Because of the importance of VSTM for everyday vision, researchers have sought to discover mechanisms that increase its capacity. Luck and Vogel (1997) showed that an efficient approach to increase the limited capacity of VSTM is by chunking isolated features into integrated objects, with each object characterized by a conjunction of several features. This

We thank Marvin Chun, Greg Davis, Andrew Hollingworth, John Henderson, Steve Luck, Dawn Morales, Dan Simons, and one anonymous reviewer for helpful comments. Correspondence should be addressed to I. R. Olson, Center for Cognitive Neuroscience, University of Pennsylvania, 3720 Walnut Street B-51, Philadelphia, PA 191046241 (e-mail: iolson@psych.upenn.edu or yuhong@wjh.harvard.edu). allows four conjunction visual objects to be held with similar ease and fidelity as four simple features (Luck \& Vogel, 1997, but see Olson \& Jiang, 2002; Wheeler \& Treisman, 2002). In addition to object-based chunking, multiple items can be remembered as a single, complex pattern rather than as several isolated items (Jiang et al., 2000; Sanocki, 2003). This study investigates how VSTM may be enhanced by a third factor: learning.

\section{Short-Term Memory and Learning}

Many cognitive activities improve their efficiency with experience: Driving becomes automatic, pianoplaying effortless, and reading much faster. Yet the memory capacity of approximately 3-4 for distinct objects is observed for infants as young as 12 months (Rose, Feldman, \& Jankowski, 2001) and is also seen in adults (Luck \& Vogel, 1997). The stability over many years of development indicates that VSTM capacity is relatively insensitive to general practice. However, specific practice, in the form of repeatedly encoding and storing in VSTM the same exact display many times, may create a visual long-term memory (VLTM) for the repeated display. In this study, we test how building a VLTM for a display may influence performance in VSTM on that display.

Unlike VSTM, VLTM has high capacity. After seeing thousands of pictures of natural scenes only once, humans can sort them out from new pictures with high accuracy (Nickerson, 1968; Standing, Conezio, \& Haber, 1970). In addition, visual details extracted from complex scenes can be retained in visual memory for minutes or even hours (Hollingworth, 2004; Hollingworth \& Henderson, 2002), allowing future performance to rely on VLTM only. Finally, extremely complex visual information, 
such as the layout of a display, can be retained in an implicit long-term memory (LTM; Chun \& Jiang, 1998, 2003). Such VLTM enhances perception: When the same display has to be processed for a second time, performance becomes more efficient, although participants are not aware of having seen the display before. Because such facilitation often occurs as a form of automatic priming, it is unclear whether VLTM can also facilitate VSTM, which requires deliberate encoding and retrieval.

In the verbal domain, short-term memory (STM) and long-term memory are often conceptualized as separate but interacting entities. Digit span can be increased by simply repeating the same list of digits-presumably forming an LTM trace-every few trials (Hebb, 1961). Other studies of verbal memory have shown that lengthy training can improve digit span from about 7 to about 80 digits (Chase \& Ericsson, 1981).

What mechanism allows VLTM to benefit VSTM? Although VLTM may not change the number of memory "slots" in VSTM, it can modulate VSTM performance by allowing more information to be chunked together, effectively increasing the "functional capacity" of VSTM. Such enhancement has been demonstrated for verbal STM, where chunking on the basis of long-term knowledge can effectively increase the total number of digits retained in verbal STM (Baddeley \& Logie, 1999; Ericsson \& Kintsch, 1995). For visual stimuli, chunking would most readily occur when items conform with Gestalt grouping cues (Jiang, Chun, \& Olson, 2004). In the absence of such cues, chunking may not occur, making it difficult for VLTM to enhance VSTM.

A second form of long-term enhancement is that VLTM may provide additional information about what can be extracted and retained in VSTM. Additional, nonvisual information includes information about the gist of the scene and conceptual knowledge about individual objects appearing in the scene. Furthermore, VLTM may provide more visual details, in which case VSTM performance may be enhanced by having information from two stores. We term this the nonredundant-representation hypothesis.

The alternative to this hypothesis is that LTM may not modulate VSTM performance, because visual information that is retained in LTM is completely redundant with - or inferior to - the information that can be extracted on the fly. We term this the redundant-representation hypothesis. For instance, one may have a representation of a constellation such as the Big Dipper (i.e., Ursa Major) in LTM, but this information can also be directly extracted after briefly viewing the Big Dipper. If one saccades away and then back to the same constellation, accuracy at reporting whether the Big Dipper has changed will be just as accurate as reporting whether a novel constellation has changed, even though one lacks a long-term representation of the other constellation. In other words, having knowledge of the Big Dipper's appearance does not improve VSTM.

In this article, we ask a straightforward question: Is VSTM performance enhanced by VLTM? To test this, we use a change-detection paradigm for locations (Experiments 1A and 1B) and novel shapes (Experiment 2). VLTM traces were created by repeatedly presenting the same displays. Evidence for the existence of VLTM was gathered at the end of the experiment by a recognition task.

\section{EXPERIMENTS 1A AND 1B}

Experiments 1A and 1B tested whether repetition of spatial locations can improve VSTM for those stimuli. Participants were required to remember the locations of several green squares (see Figure 1). Accuracy was assessed over 24 blocks of old and new displays. This number of repetitions can lead to LTM traces of the repeated spatial configurations (Chun \& Jiang, 1998). If the LTM trace enhances on-line change detection, this finding would support the nonredundant-representation hypothesis.

\section{Method}

Participants. Six participants were tested in Experiment 1A and 12 in Experiment 1B.

Materials. The stimuli were green squares $\left(0.76^{\circ}\right)$ presented on a uniformly gray background that subtended $15.6^{\circ} \times 15.6^{\circ}$ of visual angle. Locations were chosen from an invisible $10 \times 10$ matrix with 100 possible locations. The stimuli were designed so that squares could not touch one another.

Task. Each trial of the VSTM task started with a memory display lasting $500 \mathrm{msec}$, followed by a retention interval of $500 \mathrm{msec}$, and then a probe display lasting until response. The memory display contained green squares presented at randomly selected locations. The probe display contained either the same number of filled locations (Experiment 1A) or one filled location (Experiment 1B). In both cases, the critical filled location on the probe display- the one that changed (e.g., different trials) or did not change (e.g., same trials)was cued by a red outline box to assist decision making. The participants did not know which item would be probed at trial onset, so they had to encode all items on the memory display. The participants pressed one of two keys to report whether the cued location matched a location on the memory location. Upon their doing so, the screen was cleared and they were cued to initiate the next trial. The participants were not informed of the repetition across blocks. Only accuracy was emphasized.

Design. The participants were tested in 24 blocks ( 12 trials each) of VSTM trials followed by 1 block of recognition trials. In both experiments, three factors were varied: condition (old or new), set size $(6,9$, or 12 green squares), and target change (same or different). We first describe common aspects of the experiments.

The condition variable was manipulated on the memory display. Each trial within a block was a different configuration of green squares on the memory display. Six of the configurations belonged to the repeated or old condition, and each of the old configurations was presented once per block for 24 blocks. Thus, there were two instances of each set size in the old condition. The other six trials belonged to the new condition: each memory configuration was randomly generated afresh in each block. As in the old condition, there were two instances of each set size in the new condition. The old memory configurations were not predictive of whether there would be a change on the probe display or where the change would be. The old displays simply repeated their configuration on the memory display many times over the course of the experiment. New and old trials were randomly intermixed within a block.

The probe display in Experiment 1A was either identical to the memory display on $50 \%$ of the trials or differed in one location on $50 \%$ of the trials. Experiment $1 \mathrm{~B}$ assessed memory using a probe 


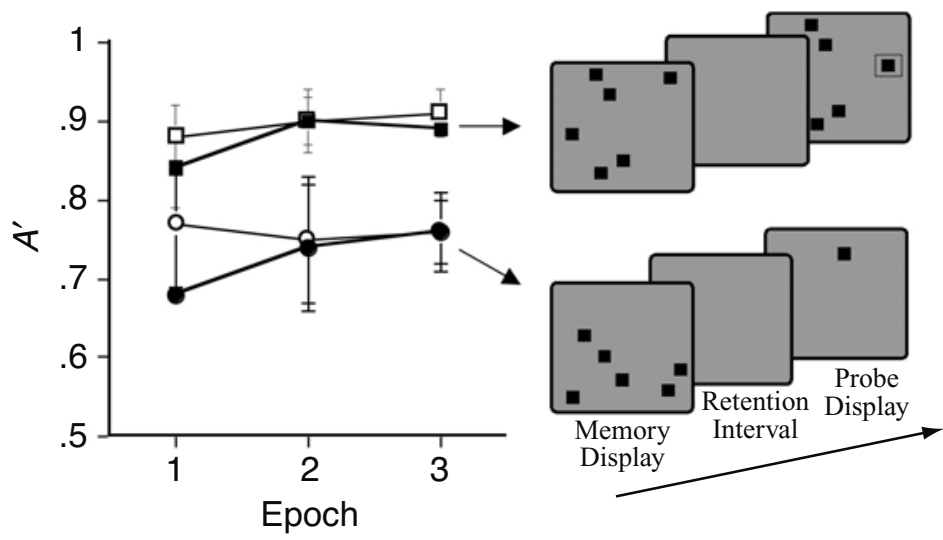

$$
\begin{aligned}
& \longrightarrow-\text { Old-Full probe } \\
& \longrightarrow \square \text { New-Full probe } \\
& \longrightarrow \text { - Old-Single probe } \\
& \longrightarrow \text { - New-Single probe }
\end{aligned}
$$

Figure 1. Results from Experiments 1A and 1B: $A^{\prime}$ as a function of condition collapsed across set size. The old condition from Experiment $1 \mathrm{~A}$ is represented by the black filled squares; the old condition from Experiment 1B is represented by the black filled circles. The new condition from Experiment $1 \mathrm{~A}$ is represented by the black unfilled squares; the new condition from Experiment $1 \mathrm{~B}$ is represented by the unfilled circles. To the right of the graph is a depiction of the stimuli and procedure used in Experiments $1 \mathrm{~A}$ and $1 \mathrm{~B}$. The actual stimuli were smaller with respect to the display than what is depicted in the figure.

display with only one filled location that was either the same as a previously filled location or different from a previously filled location. Two types of probe displays were tested, because previously we found that VSTM was more accurate for full-probe displays than for single-probe displays (Jiang et al., 2000). However, we were concerned that trials containing a changed location within a known configuration would constitute a new but similar configuration that could interfere with a veridical representation of the memory display. Single probes were tested to reduce such interference.

Recognition test. The participants completed one block of recognition test after completion of the VSTM trials. Six of the trials were old configurations, and the other six trials were new configurations, randomly intermixed. The memory display was presented until a response was made. The participants pressed the " $y$ " key to report recognition of an old display and the "n" key if they did not recognize the display.

Data analysis. $A^{\prime}$ was calculated as a measure of sensitivity. Chance performance corresponds to $A^{\prime}$ of .5 , and perfect performance to 1 .

\section{Results and Discussion}

The 24 blocks were binned into three epochs to increase power (see Table 1, Figure 1, and the Appendix). Three factors were analyzed in an analysis of variance (ANOVA): set size, epoch, and condition.

Experiment 1A: Full probe. There were significant main effects of set size $[F(2,10)=10.09, p<.004]$, showing reduced accuracy as set size increased, and epoch $[F(2,10)=4.16, p<.05]$, showing improvement of accuracy over time. However, the main effect of con- dition was not significant $[F(1,5)<1]$, suggesting that performance was not influenced by repetition of the old displays. The interaction of set size and epoch approached significance $[F(4,20)=2.40, p<.084]$, due to increased accuracy at set size 12 over time for both old and new conditions. None of the other interactions were significant (all $p \mathrm{~s}>.10)$.

Experiment 1B: Single probe. Was the lack of improvement in Experiment 1A due to interference of the (changed) probe on the memory display? The singleprobe condition reduced such interference. An ANOVA on set size, epoch, and condition revealed a main effect of set size $[F(2,18)=7.43, p<.004]$, but the effects of epoch and condition were not significant $[F(2,18)=$ $1.47, p>.25 ; F(1,9)<1]$, nor were any of the interaction effects significant (all $p \mathrm{~s}>.10$; see Table 1, Fig-

\begin{tabular}{|c|c|c|c|c|c|c|c|c|c|c|}
\hline \multirow[b]{2}{*}{ Probe } & \multirow[b]{2}{*}{ Condition } & \multicolumn{3}{|c|}{ Set Size 6} & \multicolumn{3}{|c|}{ Set Size 9} & \multicolumn{3}{|c|}{ Set Size 12} \\
\hline & & E1 & E2 & E3 & E1 & E2 & E3 & E1 & E2 & E3 \\
\hline \multirow[t]{2}{*}{ Full } & & . & .9 & .97 & 91 & .92 & .88 & .75 & .86 & .91 \\
\hline & & .94 & .9 & .9 & .95 & .9 & .93 & .79 & .84 & .87 \\
\hline \multirow[t]{2}{*}{ Single } & & .85 & .86 & .82 & .76 & .69 & .80 & .70 & .67 & .71 \\
\hline & Old & .75 & .81 & .88 & .69 & .77 & .76 & .64 & .71 & .71 \\
\hline
\end{tabular}
ure 1, and the Appendix).

Table 1

$A^{\prime}$ Observed in Experiments 1A and 1B

Note-E, epoch. Chance performance corresponds to an $A^{\prime}$ of .5, and perfect performance corresponds to an $A^{\prime}$ of 1 . 
To increase statistical power, data were pooled across the full- and single-probe conditions, and probe type was added as a between-subjects factor. There was a significant main effect of probe type $[F(1,14)=25.33, p<$ $.0001]$, with better accuracy in the full-probe than in the single-probe condition. There was also a significant main effect of set size $[F(2,28)=11.60, p<.001]$. However, this analysis did not reveal a significant effect of epoch $[F(2,28)=2.23, p>.10]$ or condition $[F(1,14)<1]$. None of the interactions involving condition were significant (all $F$ s $<1$ ).

Recognition test. In the full-probe condition, mean hit rate $(78 \%)$ and mean false alarm rate $(34 \%)$ were significantly different $[t(5)=4.33, p<.01]$. Similarly, in the single-probe condition, mean hit rate $(80 \%)$ and mean false alarm rate $(50 \%)$ were different $[t(9)=2.71, p<$ $.02]$. Thus, participants were able to recognize which displays were repeatedly presented.

To assess whether there was any relationship between explicit recognition and training-related performance changes, we conducted a correlational analysis on the data from participants in Experiment 1B because this study had the most participants. Two scores were used from each participant: the VSTM difference score was old - new accuracy for the last epoch of the VSTM session, and the recognition difference score was corrected accuracy for the old condition (e.g., hit rate - false alarm rate) in the recognition task. The correlation between the VSTM difference score in the last epoch of training and the recognition difference score was not significant $(r=-0.548, p=.10)$. It should be noted that although the magnitude of the correlation is high, it is in the opposite direction that one would need to support the hypothesized relationship between VSTM training and recognition performance. In sum, participants with better recognition scores did not show larger VSTM improvements. This suggests that long-term recognition did not facilitate VSTM performance.

These data show that in Experiment 1A, there was a general benefit of training, as illustrated by the main effect of epoch. No such effect was apparent in Experiment 1B. However, there was no specific effect of repeated configurations, shown by the nonsignificant interaction of condition and epoch, although participants could recognize the old displays at above-chance levels in the recognition task. That VLTM does not benefit VSTM performance stands in contrast to past studies that found effects of VLTM on visual search or counting speed (Chun \& Jiang, 1998; Lassaline \& Logan, 1993).

\section{EXPERIMENT 2}

Experiment 2 tested VSTM for shapes, to assess whether the surprising lack of improvement in Experiments 1A and $1 \mathrm{~B}$ was restricted to memory for spatial configurations. In the old condition, there was repetition of memory displays containing the same shapes in the same spatial organization, a procedure that has been shown to create an LTM trace that is accessible to implicit measures (Chun \& Jiang, 1998). In the new condition, shapes were randomly shuffled and displayed at new locations.

\section{Method}

Participants. There were 11 participants.

Materials and Task. Many aspects of the experiment were similar to what was used in Experiments 1A and 1B. Each display contained three, five, or seven items. The set size was reduced from that used in Experiments 1A and 1B because VSTM capacity is lower for shapes than for locations (Jiang et al., 2000). The items were randomly selected from a total of 10 novel shapes (see Chun $\&$ Jiang, 1999). They were presented at randomly selected locations on a $10 \times 10$ invisible matrix, as in Experiments $1 \mathrm{~A}$ and $1 \mathrm{~B}$ (see Figure 1B).

Each trial started with a memory display $(500 \mathrm{msec})$, followed by a retention interval $(906 \mathrm{msec})$, and a full-probe display (until response). The interstimulus interval was longer than that used in Experiments $1 \mathrm{~A}$ and $1 \mathrm{~B}$ because it was suggested that the null results of Experiments $1 \mathrm{~A}$ and $1 \mathrm{~B}$ might be due to the fact that VSTM did not have enough time to make contact with VLTM traces. One item on the probe display was cued by a red outline frame. The participants' task was to decide whether the shape inside the frame had changed. After the change-detection trials, there was a recognition task, similar to that used in Experiments 1A and 1B.

Design. Condition, set size, and target change were manipulated. The participants completed 24 blocks of the change-detection task and one block of recognition trials. Each block contained 12 trials, 6 from the old condition and 6 from the new condition. In the old condition, the same memory displays were repeatedly presented, once per block. In the new condition, shapes were randomly selected and presented at new locations. As in the previous experiments, the old display did not predict whether there would be a change and which item might change. On the probe display, when a change occurred (e.g., different trials), a new object that had not been presented among the items on the memory display replaced one of the memory objects.

\section{Results and Discussion}

Data were binned into three epochs (see Figure 2 and Table 2). An ANOVA on set size, epoch, and condition showed a significant main effect of set size $[F(2,20)=$ 53.32, $p<.001]$, with poorer performance at larger set sizes. However, the main effects of epoch and condition were not significant (both $F_{\mathrm{S}}<1$ ). None of the interaction effects were significant (all $p s>.20$ ). These data suggest that VSTM of simultaneously presented shapes was not assisted by long-term representations of the shapes or the locations.

During the recognition task, mean hit rate was $62 \%$, and mean false alarm rate was $42 \%[t(10)=3.2, p<$ .01]. An analysis showed no evidence for a significant correlation between the VSTM difference score and the recognition performance $(r=.283, p=.50)$.

These results suggest that participants acquired longterm representations of old displays because they could discriminate between old and new displays at abovechance levels on the recognition test. However, this ability did not correlate with performance on the VSTM task. Indeed, there was no evidence that VLTM facilitated change-detection performance. Chun and Jiang (1998) found that participants could show low levels of 


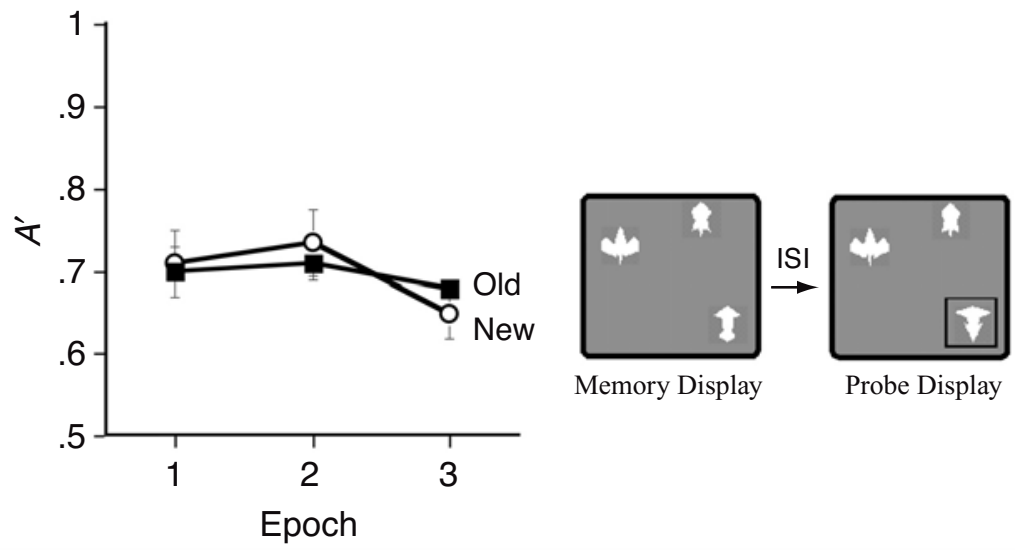

Figure 2. Results from Experiment 2: $A^{\prime}$ as a function of condition collapsed across set size. The old condition, represented by the filled squares, is the condition with the repeated configurations. The new condition, represented by the unfilled circles, is the condition with the novel, unrepeated configurations. To the right of the graph is a depiction of the stimuli used in Experiment 2. The actual stimuli were smaller with respect to the display than what is depicted in the figure.

explicit recognition and high levels of learning-related benefits on an attention task, suggesting that explicit recognition does not predict the utility or ability to benefit from VLTM traces. Our findings show an inverted similarity: high levels of explicit recognition and low levels of learning-related benefits on a short-term memory task.

\section{GENERAL DISCUSSION}

Can one hold more familiar visual information than unfamiliar visual information in VSTM? In this study, we asked whether specific knowledge (e.g., long-term memory) improved performance on a VSTM task. To create long-term memories, displays of locations or shapes were repeated many times (24) over the course of the experimental session. We proposed two competing hypotheses: (1) The nonredundant-representation hypothesis suggested that VLTM might provide a more detailed or durable representation, in which case VSTM performance should be enhanced by having information available from two stores; and (2) the redundant-representation hypothesis suggested that VLTM did not provide a more detailed or better representation so that performance on a VSTM task should not be enhanced by having information from two stores.

Table 2

$A^{\prime}$ Observed in Experiment 2

\begin{tabular}{|c|c|c|c|c|c|c|c|c|c|}
\hline \multirow[b]{2}{*}{ Condition } & \multicolumn{3}{|c|}{ Set Size 3} & \multicolumn{3}{|c|}{ Set Size 5} & \multicolumn{3}{|c|}{ Set Size 7} \\
\hline & E1 & E2 & E3 & E1 & E2 & E3 & E1 & E2 & E3 \\
\hline New & .75 & .85 & .78 & .74 & .74 & .68 & .65 & .63 & .56 \\
\hline Old & .81 & .81 & .79 & .67 & .69 & .70 & .65 & .63 & .61 \\
\hline
\end{tabular}

Note-E, epoch. Chance performance corresponds to an $A^{\prime}$ of .5, and perfect performance corresponds to an $A^{\prime}$ of 1 .
The findings showed that VSTM of locations and novel shapes was insensitive to a moderate number of repetitions that created a VLTM trace. A similar amount of practice with repeated displays is sufficient to facilitate visual search or enumeration of repeated displays (Chun \& Jiang, 1998; Lassaline \& Logan, 1993). The observed effects were not due to a failure to establish long-term representations. Indeed, our participants showed abovechance recognition of the repeated stimuli, suggesting that the repeated patterns were encoded into LTM. Although participants had memory traces of the repeated stimuli, they could not or did not use these traces to assist VSTM performance. The lack of correlation between training benefits and recognition performance further suggests that the ability to retrieve a display from VLTM does not lead to an enhanced ability to detect changes on that display.

One could argue that recognition was tested at the end of the experiment and as such, reflects the full benefit of repetition, whereas the VSTM trials did not. However, we scrutinized the data and did not find even a hint of a VSTM learning effect. In fact, our measure of recognition might have underestimated true recognition performance because the new stimuli were randomly generated and may have been quite similar to stimuli presented earlier in the experiment. Repeating the same display 24 times led to a significant visual long-term trace of the display, yet that was insufficient to enhance VSTM performance. These results have been replicated by Olson, Jiang, and Sledge (2005).

These data lend support to the redundant-representation hypothesis proposed earlier in this article. The redundantrepresentation hypothesis is made more compelling by findings showing that the resolution of VSTM is better than the resolution of VLTM. Psychophysical studies of memory for low-level visual features such as spatial fre- 
quency, orientation, and speed suggest that basic visual attributes are maintained in VSTM with great precision (Magnussen, Greenlee, \& Thomas, 1996). VSTM for high-level, realistic visual stimuli-scenes-also appears to be quite precise because subtle changes to the scene, such as a change in the orientation of a chair in a bedroom, are detected quite accurately (Hollingworth \& Henderson, 2002).

Recent findings from scene memory studies suggest that VLTM retains detailed representations of visual scenes (Hollingworth \& Henderson, 2002) such that people can remember the visual details of hundreds of individual objects (Hollingworth, 2004). It is not known whether the high fidelity of scene memory is a special case of visual memory. It is possible that simple stimuli such as colors are maintained as categorical exemplars in VLTM and lack precise details about hue and saturation, whereas stimuli with higher informational content such as items in a scene are maintained with great fidelity and as unique exemplars, due to their low degree of overlap with other stimuli. This hypothesis is reasonable because previous studies have shown that memory decay differs by stimulus type. For instance, it has been reported that memory for novel shapes and spatial frequency shows no decay over retention intervals of 8-10 sec but decay after that, whereas memory for contrast decreases linearly with retention interval (Magnussen et al., 1996; Sakai \& Inui, 2002). These findings suggest that the decay, and hence the fidelity, of memory representations are stimulus dependent. It is possible that long-term representations are less precise for simple stimuli, such as used here, than are representations for complex stimuli. If the fidelity of information held in VLTM was poor, it may have provided little benefit to VSTM.

Although the resolution of VLTM may have limited the ability of LTM to assist VSTM in the task used here, VLTM may influence VSTM performance in other tasks or when other stimuli are used. We briefly list these cases. First, LTM of conceptual and semantic information may assist VSTM for realistic stimuli. For instance, semantic properties of a scene influence how quickly a change to an item in the scene is noticed (Hollingworth $\&$ Henderson, 2000). Semantic properties of simpler displays, such as displays containing familiar faces (e.g., faces of movie stars) as compared with unfamiliar faces, may also enhance VSTM performance (Buttle \& Raymond, 2003). Second, LTM may help prioritize regions of a scene, acting as a pointer for the allocation of attention. This would occur for tasks in which foreknowledge about the location of some critical item was useful. For instance, long-term retention of the location of a target within a cluttered scene can prioritize regions of the scene for fast target localization (Chun, 2000; Chun \& Jiang, 1998; Olson, Chun, \& Allison, 2001). Contextual cuing effects are critically dependent on the invariant pairing of a target with a repeated configuration. When the repeated search displays are not correlated with a target location, there is no change in performance (Chun \&
Jiang, 1998, 1999). We now have data from a VSTM task similar to Experiment 1A showing that if the item that changes on the probe display is always the same item, display repetition will lead to an LTM trace that guides attention to the potential change, enhancing VSTM performance (Olson et al., 2005)

Our findings contrast with observations that digit span increases when the same list of digits is repeated many times (Hebb, 1961). Other studies of verbal memory have shown that lengthy training can improve digit span from about 7 to about 80 digits and was thought to result from effective chunking (Chase \& Ericsson, 1981). What accounts for this difference? In his seminal article on chunking, Miller (1956) noted that utilization of language may be the single best way to chunk information. In the case of VSTM, LTM may provide information, such as semantic knowledge or verbal associations, for chunking. This type of information may help chunk information in visual memory tasks involving familiar or semantically rich stimuli. However, overt chunking may be more difficult when the task utilizes simple visual stimuli.

In conclusion, we suggest that repetition of visual displays results in visual long-term memory but that these representations provide no benefit over and above what is established by VSTM. Future studies should address whether associative learning, as opposed to the nonassociative learning task used here, can better assist performance on VSTM tasks.

\section{REFERENCES}

Baddeley, A. D., \& Logie, R. H. (1999). Working memory: The multiple-component model. In A. Miyake \& P. Shah (Eds.), Models of working memory: Mechanisms of active maintenance and executive control (pp. 28-61). New York: Cambridge University Press.

Bor, D., Duncan, J., \& OWEn, A. M. (2001). The role of spatial configuration in tests of working memory explored by functional neuroimaging. Scandinavian Journal of Psychology, 42, 217-224.

Buttle, H., \& RAYMOND, J. E. (2003). High familiarity enhances visual change detection for face stimuli. Perception \& Psychophysics, $\mathbf{6 5}_{2}$ 1296-1306.

Chase, W. G., \& Ericsson, K. A. (1981). Skilled memory. In J. Anderson (Ed.), Cognitive skills and their acquisition (pp. 141-189). Hillsdale, NJ: Erlbaum.

Chun, M. M. (2000). Contextual cueing of visual attention. Trends in Cognitive Sciences, 4, 170-178.

CHUN, M. M., \& JIANG, Y. (1998). Contextual cueing: Implicit learning and memory of visual context guides spatial attention. Cognitive Psychology, 36, 28-71.

CHUN, M. M., \& JIANG, Y. (1999). Top-down attentional guidance based on implicit learning of visual covariation. Psychological Science, $\mathbf{1 0}$, 360-365.

CHUN, M. M., \& JIANG, Y. (2003). Implicit, long-term spatial contextual memory. Journal of Experimental Psychology: Learning, Memory, \& Cognition, 29, 224-234.

ERICSSON, K. A., \& KINTSCH, W. (1995). Long-term working memory. Psychological Review, 102, 211-245.

HeBb, D. O. (1961). Distinctive features of learning in the higher animals. In J. F. Delafresnaye (Ed.), Brain mechanisms and learning. New York: Oxford University Press.

HollingwORTH, A. (2004). Constructing visual representations of natural scenes: The roles of short- and long-term memory. Journal of Experimental Psychology: Human Perception \& Performance, $\mathbf{3 0}_{2}$ 519-537. 
Hollingworth, A., \& Henderson, J. M. (2000). Semantic informativeness mediates the detection of changes in natural scenes. Visual Cognition, 7, 213-235.

Hollingworth, A., \& Henderson, J. M. (2002). Accurate visual memory for previously attended objects in natural scenes. Journal of Experimental Psychology: Human Perception \& Performance, 28, 113-136.

IRWIN, D. (1992). Memory for position and identity across eye movements. Journal of Experimental Psychology: Learning, Memory, \& Cognition, 18, 307-317.

JiANG, Y., CHUN, M. M., \& Olson, I. R. (2004). Perceptual grouping in change detection. Perception \& Psychophysics, 66, 446-453.

JiAng, Y., Olson, I. R., \& Chun, M. M. (2000). Organization of visual short-term memory. Journal of Experimental Psychology: Learning, Memory, \& Cognition, 26, 683-702.

Lassaline, M. E., \& LoGAN, G. D. (1993). Memory-based automaticity in the discrimination of visual numerosity. Journal of Experimental Psychology: Learning, Memory, \& Cognition, 19, 561-581.

LoGIE, R. H. (1995). Visuo-spatial working memory. Hillsdale, NJ: Erlbaum.

LucK, S. J., \& Vogel, E. K. (1997). The capacity of visual working memory for features and conjunctions. Nature, 309, 279-281.

Magnussen, S., Greenlee, M. W., \& Thomas, J. P. (1996). Parallel processing in visual short-term memory. Journal of Experimental Psychology: Human Perception \& Performance, 22, 202-212.

MiLleR, G. A. (1956). The magical number seven, plus or minus two: Some limits on our capacity for processing information. Psychological Review, 63, 81-97.

NICKERSON, R. S. (1968). A note on long-term recognition memory for pictorial material. Psychonomic Science, 11, 58.

Olson, I. R., Chun, M. M., \& Allison, T. (2001). Contextual guidance of attention: Human intracranial event-related potential evidence for feedback modulation in anatomically early temporally late stages of visual processing. Brain, 124, 1417-1425.

OLSON, I. R., \& JIANG, Y. (2002). Is visual short-term memory object based? Rejection of the "strong object" hypothesis. Perception \& Psychophysics, 64, 1055-1067.

OLSON, I. R., JIANG, Y., \& SLEDGE, K. (2005). Associative learning improves visual working memory performance. Manuscript submitted for publication.

Pashler, H. (1988). Familiarity and visual change detection. Perception \& Psychophysics, 44, 369-378.

RAFFONE, A., \& WOLTERS, G. (2001). A cortical mechanism for binding in visual working memory. Journal of Cognitive Neuroscience, 13, 766-785.

Rose, S. A., Feldman, J. F., \& Jankowski, J. J. (2001). Visual shortterm memory in the first year of life: Capacity and recency effects. Developmental Psychology, 37, 539-549.

SAKAI, K., \& INUI, T. (2002). A feature-segmentation model of shortterm visual memory. Perception, 31, 579-589.

SANOCKI, T. (2003). Representation and perception of scenic layout. Cognitive Psychology, 47, 43-86.

SANTA, J. L. (1977). Spatial transformations of words and pictures. Journal of Experimental Psychology: Human Learning \& Memory, 3, 418-427.

Standing, L., Conezio, J., \& Haber, R. N. (1970). Perception and memory for pictures: Single-trial learning of 2500 visual stimuli. Psychonomic Science, 19, 73-74.

Vogel, E. K., Woodman, G. F., \& Luck, S. J. (2001). Storage of features, conjunctions, and objects in visual working memory. Journal of Experimental Psychology: Human Perception \& Performance, 27, 92-114.

WHEELER, M. E., \& TREISMAN, A. M. (2002). Binding in short-term visual memory. Journal of Experimental Psychology: General, 131, 48-64.

\begin{tabular}{|c|c|c|c|c|c|c|c|}
\hline Hits (Hs) & & $\begin{array}{r}\text { A } \\
\text { Alar }\end{array}$ & (F) & & ed & the & \\
\hline & & & Conc & & & Conc & \\
\hline Experiment & & E1 & E2 & E3 & E1 & $\mathrm{E} 2$ & E3 \\
\hline $1 \mathrm{~A}$ & $\mathrm{H}$ & .89 & .90 & .90 & .90 & .92 & .90 \\
\hline & FA & .27 & .23 & .17 & .21 & .25 & .20 \\
\hline 1B & $\mathrm{H}$ & .68 & .71 & .72 & .65 & .73 & .74 \\
\hline & FA & .30 & .34 & .30 & .37 & .32 & .30 \\
\hline 2 & $\mathrm{H}$ & .73 & .73 & .73 & .76 & .72 & .74 \\
\hline & FA & .44 & .40 & .43 & .44 & .37 & .41 \\
\hline
\end{tabular}

Note-E, epoch.

(Manuscript received January 5, 2004; revision accepted for publication March 24, 2004.) 